\title{
Deference and Uniqueness
}

\author{
Christopher J. G. Meacham \\ Forthcoming in Philosophical Studies.
}

\begin{abstract}
Deference principles are principles that describe when, and to what extent, it's rational to defer to others. Recently, some authors have used such principles to argue for Evidential Uniqueness, the claim that for every batch of evidence, there's a unique doxastic state that it's permissible for subjects with that total evidence to have. This paper has two aims. The first aim is to assess these deference-based arguments for Evidential Uniqueness. I'll show that these arguments only work given a particular kind of deference principle, and I'll argue that there are reasons to reject these kinds of principles. The second aim of this paper is to spell out what a plausible generalized deference principle looks like. I'll start by offering a principled rationale for taking deference to constrain rational belief. Then I'll flesh out the kind of deference principle suggested by this rationale. Finally, I'll show that this principle is both more plausible and more general than the principles used in the deference-based arguments for Evidential Uniqueness.
\end{abstract}

\section{Introduction}

There's been a lot of debate in the recent literature about a thesis called "Uniqueness" - or as I'll call it, Evidential Uniqueness - the thesis that, for every batch of evidence, there's a unique doxastic state that's permissible for subjects with that as their total evidence to have. Proponents of Evidential Uniqueness have offered a number of arguments in its favor, including appeals to evidential support, reliability, and the avoidance of arbitrariness. ${ }^{1}$ But these arguments have done little to sway opponents of Evidential Uniqueness. ${ }^{2}$

Recently, a couple of authors have independently advanced a new kind of argument for Evidential Uniqueness, an argument that appeals to deference considerations. ${ }^{3}$ These arguments, if correct, provide a powerful new reason to favor Evidential Uniqueness.

\footnotetext{
${ }^{1}$ For example, see White (2005) and Matheson (2011).

${ }^{2}$ For some replies, see Ballantyne \& Coffman (2011), Kelly (2013), Schoenfield (2013), Meacham (2013), Peels $\&$ Booth (2014), and Titelbaum \& Kopec (manuscript).

${ }^{3}$ The arguments I'll consider, offered by Levinstein (2015) and Greco \& Hedden (2016), appeal to a kind of deference principle in order to provide an argument for Evidential Uniqueness. Dogramaci \& Horowitz (2016) have also presented an argument for Evidential Uniqueness that appeals (in part) to considerations involving deference. But Dogramaci and Horowitz's argument is very different from the arguments offered by Levinstein and Greco and Hedden. While Levinstein and Greco and Hedden and offer theoretical arguments driven by particular deference
} 
This paper has two aims. The first is to assess these deference-based arguments for Evidential Uniqueness. I'll show that these arguments only work given a particular kind of deference principle. And I'll argue that there are independent reasons to reject these kinds of principles.

The second aim of this paper is to spell out what a plausible generalized deference principle looks like. I'll start by offering a principled rationale for taking deference to constrain rational belief. Then I'll flesh out the kind of deference principle suggested by this rationale. I'll show that this principle is both more plausible and more general than the principles used in the deference-based arguments for Evidential Uniqueness. And I'll show that this principle won't yield an argument for Evidential Uniqueness.

The rest of this paper will proceed as follows. In section two I'll sketch a way of thinking about Evidential Uniqueness that will be helpful in the discussion to come. In section three I'll discuss Greco \& Hedden's (2016) deference argument and the deference principle that it employs, and offer some reasons for being dissatisfied with this principle. In section four I'll offer a principled rationale for a different kind of deference principle. I'll show that this principle is both more plausible and more general than Greco and Hedden's principle. And I'll show that this principle won't yield an argument for Evidential Uniqueness. I'll also address a natural worry for both Greco and Hedden's principle and this principle, and argue that this worry is illusory. In section five I'll discuss Levinstein's (2015) argument and the quantitative deference principle it employs, and argue that we have reason to be dissatisfied with this principle as well. In section six, I'll briefly rehearse the morals of this discussion.

\section{Background}

\subsection{Evidential Uniqueness}

Consider any normative realm - such as the realm of moral norms, the realm of prudential norms, the realm of epistemic norms, and so on. One can think of each of these normative realms as being associated with a permission function - a function whose inputs are whatever is normatively relevant, and whose outputs are whatever is permissible. ${ }^{4}$ So the permission function associated with prudential rationality, according to standard decision theory, takes a subject's beliefs, desires, and options as inputs, and outputs the subset of these options that maximize the subject's expected desire-satisfaction. The permission function assigned to objective moral obligation, according to the Hedonic Act Utilitarian, takes a subject's options and causal facts about the hedonic consequences of those actions as inputs, and outputs the subset of those options that bring about the most hedonic utility. And the permission function corresponding to epistemic rationality, according to the extreme Subjective Bayesian, takes a subject's total evidence and background beliefs as inputs, and outputs the set of doxastic states

principles, Dogramaci and Horowitz offer an empirical argument driven by a particular application of inference to the best explanation. Thus while Dogramaci and Horowitz's argument deserves careful discussion, I won't engage with it here, since the issues it raises are largely tangential to the kind of argument I'll be focusing on.

${ }^{4} \mathrm{~A}$ function is typically defined as only having one output, whereas a permission function can intuitively have multiple outputs (if multiple things are permissible). So, strictly speaking, one might characterize the permission function as a multivalued function. Alternatively, one can take the permission function to be an ordinary function whose output is a set containing all of the permissible options. 
compatible with the canonical Bayesian norms given these inputs.

Let the epistemic permission function be the permission function associated with propositional justification - a function that takes whatever's relevant to propositional justification (for a subject at a time) as inputs, and yields whatever doxastic states are propositionally justified (for that subject at that time) as outputs. One question regarding the epistemic permission function is what its inputs are. On the one hand, someone with an exclusive view of propositional justification might take only a subject's evidence to be relevant. That is, they might endorse:

Evidential Inputs: The epistemic permission function's inputs supervene on a subject's total evidence at a time. ${ }^{5}$

On the other hand, someone with a more inclusive view of propositional justification might allow all sorts of things to be relevant - a subject's evidence, their background beliefs, their doxastic dispositions, their pragmatic concerns, and so on. Such people would endorse:

Non-Evidential Inputs: The epistemic permission function's inputs do not supervene on a subject's total evidence at a time.

Another question regarding the epistemic permission function is how much latitude there is with respect to its outputs. On the one hand, someone with a strict view of propositional justification might take a given set of inputs to always pick out a unique permissible doxastic state. That is, they might endorse:

Unique Outputs: The epistemic permission function always yields a single output.

On the other hand, someone with a more relaxed view of propositional justification might sometimes take a set of inputs to yield multiple permissible doxastic states. That is, they might endorse:

Permissive Outputs: The epistemic permission function sometimes yields multiple outputs.

The thesis typically called "Uniqueness" in the literature is the claim that for every batch of evidence, there's a unique doxastic state that it's permissible for a subject with that evidence to have. This view corresponds to the conjunction of Evidential Inputs and Unique Outputs. I'll call this view Evidential Uniqueness, to distinguish it from views which accept Unique Outputs but reject Evidential Inputs. The thesis typically called "Permissiveness" in the literature is the denial of Evidential Uniqueness. So this view corresponds to the claim that either Evidential Inputs or Unique Outputs is false.

\subsection{Some Assumptions}

Given the characterization of the epistemic permission function just sketched, a belief is permissible (in the appropriate sense) iff it's propositionally justified. Thus I'll use the terms "permissible belief" and "justified belief" interchangeably. Likewise, for the purposes of this paper, I'll use the terms "permissible belief" and "rational belief" interchangeably.

I'll assume that we can understand deontic operators as kinds of modal operators that satisfy standard deontic logic. I.e., we begin with a set of worlds, and an accessibility relation

\footnotetext{
${ }^{5}$ Thus given Evidential Inputs, the epistemic permission function can always be characterized as a function whose only input is a subject's total evidence at a time.
} 
over these worlds, where the worlds accessible to a world intuitively represent the best worlds that one can get to from that world. We require this accessibility relation to be serial; i.e., to be such that every world has some world accessible to it. Then we take a proposition $A$ to be permissible at world $w$ iff $A$ is true at some world accessible to $w$; i.e., iff $A$ is true at some best world (relative to $w$ ). Likewise, we take a proposition $A$ to be obligatory at world $w$ iff $A$ is true at every world accessible to $w$; i.e., iff $A$ is true at every best world (relative to $w$ ).

Let $P$ be the permission operator and $O$ the obligation operator. Call conditional norms of the form $A \rightarrow P(C)$ or $A \rightarrow O(C)$ "narrow scope norms", and conditional norms of the form $P(A \rightarrow C)$ or $O(A \rightarrow C)$ "wide scope norms". For the purposes of this paper I'll assume that ordinary deontic conditionals like "if $A$, then you ought to $C$ " can be understood as narrow scope norms, and ordinary deontic conditionals like "it ought to be the case that if $A$ then you $C$ " can be understood as wide scope norms. Some have defended more sophisticated ways of understanding deontic conditionals. ${ }^{6}$ But for the purposes of this paper, this simple way of understanding them is good enough.

\section{Deference}

Suppose you believe that Jane's epistemic situation is either the same as or better than yours, in every normatively relevant respect. And suppose you believe that Jane is perfectly rational, and that she believes $A$. Then it seems rational for you to believe $A$ as well. That is, it seems rational for you to defer to Jane's belief that $A$.

Recently, Greco \& Hedden (2016) have appealed to this kind of deference consideration in order to argue for Evidential Uniqueness. Greco and Hedden assert that the right principle governing rational deference is this: ${ }^{7}$

Deference: For any proposition $X$ and subject $S$, it's obligatory to be such that: if you believe $S$ 's believing $X$ is permissible, and believe you do not have any relevant evidence that $S$ lacks, then you believe $X$.

And given Deference, Greco and Hedden argue that we should accept Evidential Uniqueness, because "if two agents have the same total evidence but different beliefs about whether $P$, then

\footnotetext{
${ }^{6}$ There's a large literature on alternative ways of understanding deontic conditionals; for some recent discussions and further references, see McNamara (2010), vonFintel (2011) and Kratzer (2012).

${ }^{7}$ Greco \& Hedden (2016) formulate Deference as follows (p372-373): “...agents ought to satisfy the following conditional: Deference: If agent $S_{1}$ judges that $S_{2}$ 's belief that $P$ is rational and that $S_{1}$ does not have relevant evidence that $S_{2}$ lacks, then $S_{1}$ defers to $S_{2}$ 's belief that $P$."

A couple comments. First, placing the ought/obligatory operator outside of the statement of Deference, as Greco and Hedden do, obscures some features of Deference's normative structure that will turn out to be important. So I've moved this operator into the statement of the principle. Second, by "rational" Greco and Hedden mean "rationally permissible" (indeed, if they didn't the principle wouldn't apply to the permissive cases Greco and Hedden focus on). Since this will also turn out to be important, I've made this explicit in my statement of the principle. Third, in order to ward off some possible misunderstandings of the principle and nearby variants of it in the discussion to come, I've made the location of the quantifiers over subjects and propositions explicit in my statement of the principle. Finally, to cut down on notation and indices, I've replaced references to $S_{1}$ with "you" and $S_{2}$ with " $S$ ". This is merely to make the principle (and my discussion of it) a bit more readable; the claims Deference (and its relatives) make about about "you" should be understood as claims that apply to all subjects.
} 
you cannot defer to each's belief about whether $P$ on pain of inconsistency." ${ }^{8}$

I'll assess this argument in two stages. First, in section 3.1, I'll present the valid argument that I think best fits Greco and Hedden's remarks, and I'll flag some implicit assumptions that this argument requires. Then, in section 3.2, I'll raise some worries regarding Deference and its role in this argument.

\subsection{The Argument}

Greco \& Hedden (2016) argue that if Evidential Uniqueness is false then Deference threatens to yield inconsistent deferential commitments. Greco and Hedden don't spell out this argument in much detail. But I take this to be the valid argument that best fits their remarks: ${ }^{9}$

P1. If it can be obligatory to believe both that $S$ has the same evidence as you and permissibly believes $X$, and that $S^{*}$ has the same evidence as you and permissibly believes $\neg X$, then it can be obligatory to both believe $X$ and $\neg X$.

P2. It can't be obligatory to both believe $X$ and $\neg X$.

L1. Therefore, it can't be obligatory to believe both that $S$ has the same evidence as you and permissibly believes $X$, and that $S^{*}$ has the same evidence as you and permissibly believes $\neg X$. [From P1, P2.]

P3. If Evidential Uniqueness is false, then it can be obligatory to believe Evidential Uniqueness is false.

P4. If it can be obligatory to believe Evidential Uniqueness is false, then it can be obligatory to believe both that $S$ has the same evidence as you and permissibly believes $X$, and that $S^{*}$ has the same evidence as you and permissibly believes $\neg X$.

C. Therefore, Evidential Uniqueness isn't false. I.e., Evidential Uniqueness is true. [From P3, P4, L1.]

This argument for Evidential Uniqueness relies on four premises, so let's briefly look at each of them. P1 is a consequence of Deference. For if it's obligatory to believe that $S$ has the same evidence as you and permissibly believes $X$, then Deference entails that it's obligatory for you to believe $X$. And if it's also obligatory to believe that $S^{*}$ has the same evidence as you and permissibly believes $\neg X$, then Deference entails that it's also obligatory for you to

${ }^{8}$ Greco \& Hedden (2016), p373.

${ }^{9}$ Greco \& Hedden (2016) present the argument as follows:

“combining Permissivism with Deference... threatens to yield inconsistent deferential commitments. ... In a nutshell, if two agents have the same total evidence but different beliefs about whether $P$, then you cannot defer to each's belief about whether $P$ on pain of inconsistency. Let's take this a bit more slowly. Suppose you know that one agent has credence $n$ in $P$ while another has credence $m$ in $P$, and that they have the same total evidence. Then, you cannot defer to each one's credence in $P$; you cannot simultaneously adopt credence $n$ in $P$ and credence $m$ in $P$, where $n \neq m$. Hence, if judging that an agent's credence is rational involves a commitment to deferring to that agent's credence, then you cannot judge each agent's credence in $P$ to be rational." (p373) 
believe $\neg X$. So given Deference, we should accept P1. I'll postpone the question of whether we should accept Deference itself to section 3.2.

P2 follows from the assumption that it's impossible to both believe $X$ and $\neg X$, and the assumption that there are no epistemic dilemmas - no situations in which every possible doxastic state is impermissible. For if it's impossible to both believe $X$ and $\neg X$, then the obligation to believe both $X$ and $\neg X$ puts you in an epistemic dilemma. And if there are no epistemic dilemmas, then it follows that you can't be obligated to believe both $X$ and $\neg X$. Since I take both of these assumptions to be prima facie plausible, I'll grant $\mathrm{P} 2$ in what follows.

I take P3 to be motivated by the idea that there are situations in which it's obligatory to believe the truth of the matter with respect to whether Evidential Uniqueness is true or not. For example, if Evidential Uniqueness is true, perhaps there are situations in which one is presented with evidence or arguments that are strong enough to make it obligatory to believe Evidential Uniqueness. While one might contest this - perhaps certain a priori claims (e.g., Goldbach's conjecture) are epistemically elusive enough so as to make it always permissible to believe either it or its negation, and perhaps Evidential Uniqueness is one of those claims I'll also grant P3 in what follows.

P4 might almost seem like a logical truth. But there are coherent views on which P4 is false, such as a view on which it can be obligatory to believe Evidential Uniqueness is false (perhaps because there can be situations in which you're presented with compelling evidence or arguments to this effect), and yet on which it can't be obligatory to believe there are subjects $S$ and $S^{*}$ with the same evidence as you who permissibly believe $X$ and $\neg X$ (perhaps because certain kinds of indexical evidence make it impossible for different subjects to have the same total evidence). That said, I think many opponents of Evidential Uniqueness would accept P4, and I will grant P4 in what follows.

\subsection{Worries about Deference}

The argument for Evidential Uniqueness presented in section 3.1 hangs on the truth of P1, which is entailed by Deference. But there are several reasons to be dissatisfied with Deference, and with its role in this argument.

Perhaps the biggest reason to be dissatisfied with Deference is that its "mismatching" deontic operators make it implausible. ${ }^{10}$ Leaving Deference's "believe you have no more evidence" clause and its reference to $S$ implicit, Deference effectively says that, for any proposition $X$, it's obligatory to be such that you believe $X$ if you think it's permissible to believe $X$. But it seems implausible to maintain that it's obligatory to believe what you think is permissible after all, you might think it's permissible to believe any one of several incompatible things.

Another way to see how strange Deference's mismatching operators are is to consider the analog of such a principle in other contexts. Consider, for example, the prudential analog of Deference:

${ }^{10}$ Of course, what's problematic is not merely that we've formulated Deference using two different deontic operators. Since the permission and obligation operators are inter-definable, it's trivial to reformulate any principle with two deontic operators so that it employs only permission or obligation operators. What's problematic is that the amount of latitude in what the principle demands of the subject (in cases of deference) doesn't line up with the amount of latitude that the subject believes to obtain (in these cases of deference). 
Deference (prudential): For any act $X$ and subject $S$, it's obligatory to be such that: if you believe $S$ 's doing $X$ is permissible, and believe the normatively relevant facts are the same for you and $S$, then you do $X .^{11}$

Suppose you must take a bet on a fair coin toss at even odds, and are deciding whether to pick heads or tails. It seems you could permissibly believe that $S$ and $S^{*}$ are in the same situation (and that all of the normatively relevant facts are the same), and that $S$ permissibly picks heads while $S^{*}$ permissibly picks tails. But then Deference (prudential) would entail that you're obligated to both pick heads and tails. Few would accept such a principle. ${ }^{12}$

A proponent of Unique Outputs - like anyone who accepts Evidential Uniqueness - might be tempted to reply to these worries as follows:

Unique Outputs is true. And you should believe it's true. And if you should believe Unique Outputs is true, then you should believe that anything that's permissible is obligatory. But then to maintain (as Deference does) that it's obligatory to believe what you think is permissible is just to maintain that it's obligatory to believe what you think is obligatory. And it's plausible to maintain that it's obligatory to believe what you think is obligatory. So Deference's mismatching operators don't make it implausible after all.

This reply is unsatisfactory. Suppose it's true that you should believe Unique Outputs. As the reply sketched above notes, a principle like Deference (which maintains that it's obligatory to believe what you think is permissible) will yield the same prescriptions as a variant of Deference - call it Deference* - with matching obligation operators (i.e., which maintains that it's obligatory to believe what you think is obligatory). But Deference* is strictly more attractive

\footnotetext{
${ }^{11}$ Deference applies to both cases in which (a) you believe $S$ is in the same epistemic situation (has the same evidence), and (b) cases in which $S$ is in a better epistemic situation (has strictly more evidence). But I've restricted Deference (prudential) to just apply to the analog of the (a) cases - cases in which (you believe) you're both in the same prudential situation. I've done this because, in the context of prudential assessments, it's difficult to see what kind of fact would put one subject in a better prudential situation than another. In any case, it doesn't matter; this special case is enough to show how a norm like Deference which employs mismatching deontic operators is implausible in prudential contexts.

${ }^{12}$ In section 3 of their paper, Greco \& Hedden (2016) discuss the analog of Unique Outputs in prudential contexts, and defend the viability of such a principle. Such a principle seems difficult to maintain in the kind of coin toss case described in the text. Let the prudential permission function be a function which takes a subject's credences, utilities and available options as inputs, and yields the permissible subset of those options as outputs. Then the following two claims:
}

(1) Expected Utility Maximization: an option is prudentially permissible iff it maximizes expected utility.

(2) Existence of Symmetric Cases: there are cases in which more than one option maximizes expected utility (such as the coin toss case described above).

entail that there are cases in which multiple options are prudentially permissible. Thus adopting the prudential analog of Unique Outputs with respect to options requires rejecting either (1) or (2). Neither choice seems attractive.

What would Greco and Hedden say about the coin toss case? In correspondence, Greco and Hedden have suggested a couple potential replies. First, they might grant that the prudential analog of Unique Outputs with respect to options is false, but maintain that a prudential analog of Unique Outputs with respect to preferences is true. And they might claim that permissions regarding preferences are more fundamental than permissions regarding options. Second, they might accept the prudential analog of Unique Outputs with respect to options and reject (1), by holding that in symmetric cases like the coin toss case neither option is prudentially permissible. 
than Deference. For if you should believe Unique Outputs, then Deference* is indistinguishable from Deference. And if it's not the case that you should believe Unique Outputs, then

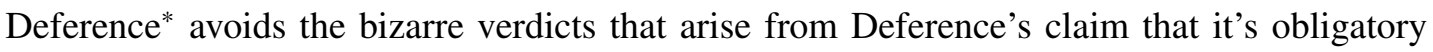
to believe whatever you think is permissible. Thus there's no reason to favor Deference over Deference*.

And in any case, it's problematic to offer this defense of Deference while using Deference in an argument for Evidential Uniqueness. For in the context of an argument for Evidential Uniqueness - a thesis with Unique Outputs as one of its conjuncts - defending the argument by assuming that one should believe Unique Outputs is question begging.

A second reason to be dissatisfied with Deference is that it presupposes Evidential Inputs. Recall what Deference says:

Deference: For any proposition $X$ and subject $S$, it's obligatory to be such that: if you believe $S$ 's believing $X$ is permissible, and believe you do not have any relevant evidence that $S$ lacks, then you believe $X$.

Deference tells us that the only feature of $S$ 's situation that we need to pay attention to is their evidence, presumably because their evidence is the only thing that's normatively relevant. But there's no reason to make such a restriction - there's no obstacle to formulating a deference principle in a way that's neutral about what the inputs are by replacing talk of evidence with talk of whatever facts are normatively relevant (cf. section 4).

Now, Greco \& Hedden (2016) offer an argument against variants of Deference which take factors other than evidence, such as "epistemic standards", into consideration. Their main complaint about such principles is that they "break the link between deference and judgments of rationality" (Greco \& Hedden (2016), p375), because they don't tell you to defer to any belief you think is rationally held simpliciter, they only tell you to defer to any belief you think is rationally held by a subject with the same epistemic standards. But this criticism applies just as much to Deference as it does to the alternatives they consider. For Deference doesn't tell you to defer to any belief you think is rationally held simpliciter, it only tells you to defer to beliefs you think are rationally held by subjects with at least as much evidence.

More generally, no tenable deference principle will provide the kind of direct link between judgments of rationality and deference that Greco and Hedden are asking for. For every tenable deference principle will grant that there are cases in which you should judge someone to be rational and yet not defer to them (e.g., because you know they have less evidence than you).

In any case, it's problematic to employ a principle like Deference that presupposes Evidential Inputs in an argument for Evidential Uniqueness. For in the context of an argument for Evidential Uniqueness - a thesis with Evidential Inputs as one of its conjuncts - presupposing Evidential Inputs is question begging.

A third reason to be dissatisfied with Deference stems from how specific the principle is. Deference only attempts to capture the rational import of deference in the epistemic realm. But the intuitions underlying deference principles seem more general than this, since the same kinds of intuitions seem to arise in prudential and moral realms as well. E.g., the intuition that "believing that someone in your epistemic situation should believe $X$ affects what you should believe" seems to stem from the same source as the intuition that "believing that someone in your moral situation should do $X$ affects what you should do". Given this, it would be more satisfying if one could provide a deference principle that applies to any normative realm, and 
which yields the appropriate deference principle in the epistemic realm as a special case.

Moreover, because of Deference's mismatching operators, Deference can't be plausibly generalized in this way. I.e., suppose we generalize Deference to any normative realm, by characterizing it in terms of generic permissions instead of permissions to believe. This generalized version of Deference will entail principles like Deference and the prudential analog of Deference mentioned above as a special case. But as we've seen, principles like the prudential analog of Deference yield absurd results because of their mismatching operators. And since the generalized version of Deference entails these principles, it will yield these absurd results as well.

A fourth reason to be dissatisfied with Deference is that the form of the principle seems largely unmotivated. As we've seen, the argument for Evidential Uniqueness hangs crucially on the deference principle taking a very particular form, with mismatching deontic operators and an exclusive focus on the evidential features of a subject's situation. But Greco and Hedden do little to motivate formulating the principle this way. It would be more satisfying to maintain that Deference is the right principle governing rational deference if one could provide a principled rationale for why a deference principle should take this form.

\section{General Deference}

We want a deference principle for which we can provide a principled rationale. And we want a deference principle that's appropriately general - one which isn't restricted to just the epistemic realm. Let's look at how we might go about building such a principle.

Consider the permission function $f$ of some normative realm - the function that takes whatever's normatively relevant as inputs, and spits out whatever's permissible as outputs. If two subjects are in the same situation with respect to what's normatively relevant - i.e., the inputs to $f$ are the same for both subjects - then the outputs of $f$ will be the same for both subjects as well:

Same Inputs: If $S_{1}$ and $S_{2}$ are the same with respect to the inputs of $f$, then they'll be the same with respect to the outputs of $f$-i.e., the same things will be permissible.

Note that so far we haven't said anything contentious - Same Inputs simply follows from the definition of $f$.

Now, let an akratic subject be a subject whose beliefs or behavior fails to line up with their beliefs about what their beliefs or behavior should be. Many find it plausible that normatively ideal subjects aren't akratic. ${ }^{13}$ Thus, for example, many find it plausible that epistemically ideal subjects won't both believe $X$ and believe that they shouldn't believe $X$. Likewise, many find it plausible that prudentially ideal subjects won't both do $X$ and believe that they shouldn't do $X$. We can express this thought as follows: ${ }^{14}$

\footnotetext{
${ }^{13}$ There's a large literature on this topic; for a classic account of the irrationality of akrasia, see Davidson (1970). For some recent defenses of the irrationality of akrasia in the epistemic case, see Horowitz (2014) and Greco (2014). For a broad discussion of these issues in prudential and moral cases, and further references, see Stroud (2014).

${ }^{14}$ These two principles are wide scope, but one might take the narrow scope versions of these principles - that if you believe $X$ is permissible/obligatory for you, then $X$ is permissible/obligatory for you - to also be prima facie plausible to those sympathetic to subjectivist accounts. Plausible or not, such principles cannot be endorsed by proponents of
} 
Anti-Akratic Principle (Permission): For any proposition $X$, it's permissible to be such that if you believe $X$ is permissible, then you $X$.

Anti-Akratic Principle (Obligation): For any proposition $X$, it's obligatory to be such that if you believe $X$ is obligatory, then you $X$.

There are some normative realms for which we might think the notions of permission and obligation require an "objectivist" theory - a theory whose prescriptions aren't mediated by a subject's doxastic state. For such realms, we'll take these anti-akratic principles to be implausible. For example, suppose we're Objective Hedonic Act Utilitarians, and hold that subjects should do whatever actually brings about the most hedonic utility (regardless of the subject's beliefs about what moral theory is correct or what acts maximize utility). Then we'll take these anti-akratic principles to be implausible in the moral realm. But if we restrict our attention to normative realms for which we think the notions of permission and obligation require a "subjectivist" theory - a theory whose prescriptions are mediated by a subject's doxastic state then many will take these anti-akratic principles to be plausible. From now on, let's restrict our discussion to these kinds of "subjectivist" normative realms.

Given the anti-akratic principles just described, we can derive the following deference principle (where I've merged the permission and obligation versions for conciseness): ${ }^{15}$

General Deference (Same Inputs): For any $X$ and subject $S$, it's permissible [obligatory] to be such that: if you believe that it's permissible [obligatory] for $S$ to $X$, and believe that the normatively relevant facts are the same for you and $S,{ }^{16}$ then you $X$.

This principle is plausible, but it's also limited. For General Deference (Same Inputs) only applies to cases in which subjects are the same with respect to what's normatively relevant. It doesn't apply to cases in which a subject is in a strictly better position with respect to something that's normatively relevant (e.g., a subject has strictly more evidence than you).

Let's look at how one might extend General Deference (Same Inputs) to cover these cases as well. To begin, let's divide the inputs a permission function $f$ takes into two kinds. First, there are inputs whose values are naturally associated with a partial ordering that reflects when one of these values is strictly better than another - call these ranked inputs. An example of a ranked input might be a subject's evidence, where the partial ordering relation mirrors the entailment relation between bodies of evidence. Second, there are inputs whose values aren't naturally ordered in this way - call these unranked inputs. An example of an unranked input might be a subject's practical interests, given a view on which a subject's practical interests bear on what they should believe. ${ }^{17}$

Evidential Uniqueness. For these narrow scope principles are incompatible with Evidential Uniqueness - they entail that two subjects with the same evidence but different beliefs can be obligated/permitted to believe different things. Since I want to motivate a deference principle in a manner that's neutral with respect to Evidential Uniqueness, I won't appeal to principles like this.

${ }^{15}$ For the derivation, see the appendix. One might wonder why an appendix is needed, since General Deference (Same Inputs) might seem to follow trivially from the anti-akratic principles given above. But while the derivation of the narrow scope version of General Deference (Same Inputs) from narrow scope versions of the anti-akratic principles is straightforward, the derivation of the actual (wide scope) version of General Deference (Same Inputs) from the actual (wide scope) versions of the anti-akratic principles take a bit more time.

${ }^{16}$ I.e., believe that the inputs of $f$ are the same for you and $S$.

${ }^{17}$ For examples of such views, see Stich (1993), Fantl \& McGrath (2009) and Podgorski (2016). 
Now, if you believe $S$ is different with respect to some unranked input of $f$, there's no reason for you to defer to the prescriptions you believe apply to $S$. Likewise, if you believe $S$ is different with respect to some ranked input of $f$, and believe you aren't lower ranked with respect to this input, there's no reason for you to defer to the prescriptions you believe apply to $S$. But if you believe $S$ is higher ranked with respect to some ranked input of $f$, and the same with respect to all other inputs, then it seems you do have reason to defer to the prescriptions you believe apply to $S$.

Thus consider a view on which one's epistemic obligations are determined by one's evidence and one's practical interests. Given such a view, if you believe $S$ has different practical interests than you, there's no reason to defer to what you believe is permissible/obligatory for $S$. Likewise, if you believe $S$ has different (but not strictly more) evidence, there's no reason to defer to what you believe is permissible/obligatory for $S$. But if you believe $S$ has strictly more evidence, and is otherwise in the same epistemic situation, then you do have reason to defer to what you believe is permissible/obligatory for $S$.

Extending General Deference (Same Inputs) to take this into account yields: ${ }^{18,19}$

\footnotetext{
${ }^{18}$ I've followed Greco and Hedden here in formulating General Deference in terms of belief instead of more finegrained doxastic attitudes, like credence. And if we restrict our attention to the epistemic realm, it's natural to extend General Deference so that it takes these more fine-grained attitudes into account, allowing it to model cases in which (say) you're uncertain about what $S$ 's normative situation is, or uncertain about what it's permissible/obligatory for $S$ to believe in a situation. But it's hard to provide this kind of quantitative formulation of General Deference if we want it apply to other normative realms as well (e.g., prudential, moral, etc.) as I do. The reason is that in these other normative realms, the objects of deference (e.g., acts) aren't fine-grained in the way that credences are. And when the objects of deference aren't fine-grained, there's no easy way to turn fine-grained differences in your credence about (say) what $S$ 's normative situation is, or what it's permissible/obligatory for $S$ to believe in a situation, into finegrained differences in the verdicts the principle delivers. (That said, I talk more about the bearing of this discussion on quantitative epistemic deference principles in section 5.)

${ }^{19}$ The prescriptions of General Deference will depend, in part, on which inputs one takes to be ranked inputs, and what one takes the partial ordering associated with these ranked inputs to be. And while the answers to these questions are relatively straightforward in some cases (e.g., it's natural to take evidence to be a ranked input with a partial ordering determined by entailment), there are other cases in which there are substantive questions to be settled, questions that will impact the prescriptions General Deference makes.

For example, consider a view which takes background beliefs to be an input, and holds that only some background beliefs are permissible. One stance, given such a view, is that background beliefs are ranked inputs, and that all permissible background beliefs are ranked higher than all impermissible background beliefs. A second stance is that background beliefs are ranked inputs, but only some particular permissible background beliefs are ranked higher than some particular impermissible background beliefs. (For example, if some impermissible background beliefs $B$ are almost rational - if one added a certain belief, they would be permissible - then one might take the permissible background beliefs you get by adding that belief to $B$ to be higher ranked than $B$. But for other permissible background beliefs, such as ones that assign radically different beliefs than $B$, there might be no ranking between them and $B$.) $\mathrm{A}$ third stance is that background beliefs are unranked inputs, because while some of them (e.g., the permissible ones) are better in some respects than some of the others (e.g., the impermissible ones), none of them are strictly better in all respects.
}

All three of these stances are compatible with General Deference. And General Deference will make somewhat different prescriptions depending on which of these stances one adopts. (If one likes, one can think of these different stances about what inputs are ranked inputs, and what their partial orders are, as something that's part of the normative principle. On this way of thinking about things, each of these different stances yields a different version of General Deference. And one can take the case made in the text for adopting General Deference as an argument for a principle of this general form, an argument that leaves open the further question of which particular version of General Deference 
General Deference: For any $X$ and subject $S$, it's permissible [obligatory] to be such that: if you believe that it's permissible [obligatory] for $S$ to $X$, and believe the normatively relevant facts are either the same for you and $S$, or differ only regarding normatively relevant facts for which $S$ is in a strictly better position, ${ }^{20}$ then you $X$.

General Deference, like Deference, is a principle that governs rational deference. But General Deference is more attractive than Deference in a number of respects. First, there is no odd mismatch in the deontic operators General Deference employs. Second, General Deference doesn't build in any potentially question begging assumptions about what's normatively relevant. Third, General Deference isn't restricted to the epistemic realm - it applies just as well to the prudential and moral realms. Fourth, we've seen a principled rationale for adopting something like General Deference, and for thinking that it takes the form that it does. ${ }^{21}$

General Deference can also explain why Greco and Hedden found Deference appealing. If we assume Evidential Uniqueness is true, and that the subject knows this, General Deference and Deference will make the same prescriptions in the epistemic case. Given Unique Outputs, something is epistemically permissible iff it's epistemically obligatory, and so it doesn't matter whether the deontic operators match. And given Evidential Inputs, the only factor normatively relevant to epistemic permission is a subject's evidence, so there's no harm in building this assumption into one's deference principle, as Deference does.

So General Deference is strictly more attractive than Deference. But General Deference won't yield an argument for Evidential Uniqueness. The main reason for this is that the Deference-based argument for believing Evidential Uniqueness crucially relies on the fact that it employs mismatching deontic operators.

To see this, recall how the first half of the Deference-based argument for believing Evidential Uniqueness goes. (To simplify the discussion, I'll leave Deference's "believe you have no more evidence" clause and its reference to $S$ implicit.) Roughly, Deference asserts that, for all $X$, the following conditional is obligatory (true at all best worlds): if you think it's permissible to believe $X$, then you believe $X$. So if there is a best world $w$ at which you think that it's both permissible to believe $X$ and permissible to believe $\neg X$, then these conditionals entail that at $w$ you believe $X$ and believe $\neg X$, which is impossible. Thus there's no best world at which you both think it's permissible to believe $X$ and permissible to believe $\neg X$. I.e., it's impermissible to think it's both permissible to believe $X$ and permissible to believe $\neg X$.

Now, if one adopted a variant of Deference that employed matching permission operators (as the permission version of General Deference does), then it would only assert that, for any $X$, the following conditional is permissible (true at some best world): if you think it's permissible to believe $X$, then you believe $X$. But given this, it is unproblematic if there's a best world $w$ at which you think it's both permissible to believe $X$ and permissible to believe $\neg X$. For this principle doesn't entail that both of the conditionals "if you think it's permissible to believe

\footnotetext{
one should adopt.)

${ }^{20}$ I.e., believe that $S$ is the same with respect to the inputs of $f$, or only differs with respect to inputs for which $S$ is higher ranked than you.

${ }^{21}$ In addition to the the ways just described, General Deference is also more general than Deference in another, more subtle, way. Deference only makes prescriptions regarding cases in which you believe it's permissible for $S$ to believe $X$ and you believe $S$ actually believes $X$. General Deference doesn't require anything like the second clause in the epistemic case, it doesn't require you to believe that $S$ actually believes $X$. So this is yet another way in which General Deference is more general than Deference.
} 
$X$, then you believe $X$ " and "if you think it's permissible to believe $\neg X$, then you believe $\neg X$ " hold at $w$, just that each holds at some best world or other.

Likewise, if one adopted a variant of Deference that employed matching obligation operators (as the obligation version of General Deference does), then it would assert that, for any $X$, the following conditional is obligatory (true at all best worlds): if you think it's obligatory to believe $X$, then you believe $X$. But given this, it is again unproblematic if there's a best world $w$ at which you both think it's permissible to believe $X$ and permissible to believe $\neg X$. For this principle doesn't entail anything about what happens when you believe something is (merely) permissible.

We have a number of reasons to think that General Deference, not Deference, is the correct form of the principle that governs rational deference. And General Deference is neutral with respect to the Evidential Uniqueness debate. So there's little reason to think that these kinds of deference considerations bear on whether one should adopt Evidential Uniqueness. ${ }^{22}$

\subsection{An Objection}

Before we move on, it's worth addressing a natural worry that one might raise for both Greco and Hedden's Deference principle and the General Deference principle presented in the last section. To simplify things, I'll first present it as a worry for Deference, and show how the proponent of Deference should respond to this worry. Then I'll briefly note how the same worry (and the same reply) arises for General Deference as well.

Consider the following case:

Ann and Beth: You're rational. You believe that Ann has strictly more evidence than you, and that Ann permissibly believes $X$. And you believe that Beth has strictly more evidence than Ann, and that Beth permissibly believes $\neg X$.

Since you're rational, and believe that Ann permissibly believes $X$ and has strictly more evidence than you, Deference entails that you believe $X$. And since you're rational, and believe that Beth permissibly believes $\neg X$ and has strictly more evidence than you, Deference also

\footnotetext{
${ }^{22}$ In section 3, we saw Greco \& Hedden (2016) argue that considerations regarding deference give us reason to adopt Evidential Uniqueness. In a similar fashion, Greco \& Hedden (2016) argue that considerations regarding planning give us reason to adopt Evidential Uniqueness. Their planning argument faces the same worries are their deference argument, so I'll just briefly rehearse it here. Greco and Hedden assert that the right principle governing rational planning is this (this is the more plausible of the two disambiguations they consider; see their footnote 22):
}

Planning: For any propositions $X$ and $E$, it's obligatory to be such that: if you believe that believing $X$ is permissible given evidence $E$, then you plan to believe $X$ given evidence $E$.

Greco and Hedden argue that opponents of Evidential Uniqueness who accept Planning are led to absurd results. But Planning is implausible and/or unsatisfying in many of the same ways as Deference: it employs mismatching deontic operators, it presupposes Evidential Inputs, it's arbitrarily constrained to the realm of epistemic norms, and so on. And like Deference, Planning is easily replaced with a more general principle without these demerits:

General Planning: For any $X$ and $Y$, it's permissible [obligatory] to be such that: if you believe that believing $X$ is permissible [obligatory] given normatively relevant factors $Y$ (i.e., believe that given inputs $Y$, one of $f$ 's outputs [ $f$ 's only output] is a doxastic state which believes $X$ ), then you plan to believe $X$ given normatively relevant factors $Y$.

And General Planning is neutral with respect to the Evidential Uniqueness debate. 
entails that you believe $\neg X$. So Deference entails that you rationally believe $X \wedge \neg X$. But, of course, it's never rational to believe $X \wedge \neg X$. So this case seems to provide us with a reductio of Deference.

To simplify the following discussion, let $\operatorname{Def}_{\text {Ann }}$ stand for the proposition expressing your beliefs about the case that (given Deference and your rationality) entail you defer to Ann:

$\operatorname{Def}_{\text {Ann }}$ : Ann has total evidence $E \wedge F$ (where $E$ is your total evidence), and Ann permissibly believes $X .^{23}$

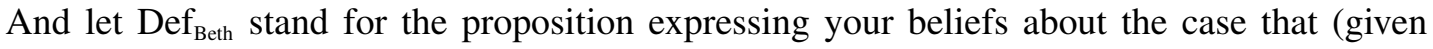
Deference and your rationality) entail you defer to Beth:

Def $_{\text {Beth }}$ : Beth has total evidence $E \wedge F \wedge G$ (where $E$ is your total evidence), and Beth permissibly believes $\neg X$.

We can diffuse this apparent reductio of Deference by noting two things. First, Deference only yields the absurd result described above if, as the description of the case requires, it's permissible to believe $\operatorname{Def}_{\mathrm{Ann}} \wedge \operatorname{Def}_{\text {Beth }}$. Second, it's plausibly impermissible to believe $\operatorname{Def}_{\text {Ann }} \wedge \operatorname{Def}_{\text {Beth }}$. Together, these two points entail that the Ann and Beth case fails to provide a reductio of Deference because the situation it describes is impossible.

Let's go through each of these two points more carefully. First, let's look at why Deference only yields this absurd result if it's permissible to believe $\operatorname{Def}_{\text {Ann }} \wedge \operatorname{Def}_{\text {Beth }}$. Deference is a wide scope norm, a norm that yields conditionals of the form $O(A \rightarrow C)$. In the example described above, the two relevant conditionals are:

(1) It's obligatory to be such that if $\left[A_{1}=\right.$ you believe $\left.\operatorname{Def}_{\text {Ann }}\right]$ then $\left[C_{1}=\right.$ you believe $\left.X\right]$.

(2) It's obligatory to be such that if $\left[A_{2}=\right.$ you believe $\left.\operatorname{Def}_{\text {Beth }}\right]$ then $\left[C_{2}=\right.$ you believe $\left.\neg X\right]$.

In order for (1) and (2) to yield the absurd result that the actual world is a best world at which both $C_{1}$ and $C_{2}$ are true (i.e., the absurd result that you rationally believe $X \wedge \neg X$ ), it needs to be the case that (a) $A_{1}$ and $A_{2}$ are actually true, and (b) that the actual world is a best world (and so, given (1) and (2), a world at which $A_{1} \rightarrow C_{1}$ and $A_{2} \rightarrow C_{2}$ hold). And in order for $A_{1}$ and $A_{2}$ to be true at the actual best world, it must be permissible to believe both $\operatorname{Def}_{\text {Ann }}$ and $\operatorname{Def}_{\text {Beth }}$. (If it was impermissible to believe both $\operatorname{Def}_{\mathrm{Ann}}$ and $\operatorname{Def}_{\text {Beth }}$, then there would be no best world at which $A_{1}$ and $A_{2}$ are true.) Thus in order for Deference to yield the absurd result that both $C_{1}$ and $C_{2}$ are true at the actual best world, it must be permissible to believe $\operatorname{Def}_{\mathrm{Ann}} \wedge \operatorname{Def}_{\text {Beth }}$.

Second, let's look at why it's plausibly impermissible to believe $\operatorname{Def}_{\text {Ann }} \wedge \operatorname{Def}_{\text {Beth }}$. Let's suppose for reductio that your total evidence justifies you in believing $\operatorname{Def}_{\mathrm{Ann}} \wedge \operatorname{Def}_{\text {Beth }}$ (i.e., justifies you in believing that Ann has strictly more evidence than you and that she permissibly believes $X$, and that Beth has strictly more evidence than both you and Ann, and that she permissibly believes $\neg X$ ). Since Ann has all of your evidence, it would seem that Ann is also justified in believing $\operatorname{Def}_{\mathrm{Ann}} \wedge \mathrm{Def}_{\text {Beth }}$. And in particular, it would seem Ann is justified in believing that Beth has strictly more evidence than her, and that Beth believes $\neg X$. But then Deference will entail that Ann should believe $\neg X$, and thus that Ann can't permissibly believe

\footnotetext{
${ }^{23}$ One might worry that if your evidence justifies you in believing that Ann has evidence $E \wedge F$, then you also have evidence $F$. And this would seem to be in tension with your belief that Ann has strictly more evidence than you. But this worry only arises if we think of $F$ as a de dicto description of Ann's additional evidence instead of a de re description of Ann's additional evidence. And we should be thinking about $F$ as a de re description of Ann's evidence.
} 
$X$. So it seems you're not justified in believing that Ann permissibly believes $X$, and thus not justified in believing Def $_{\text {Ann }}$, after all. ${ }^{24}$

Since this counterexample to Deference only works if it's permissible to believe $\operatorname{Def}_{\text {Ann }} \wedge \operatorname{Def}_{\text {Beth }}$, and it's plausibly impermissible to believe $\operatorname{Def}_{\text {Ann }} \wedge \operatorname{Def}_{\text {Beth }}$, this counterexample to Deference doesn't work. So while the Ann and Beth example raises a natural worry for Deference, this worry is ultimately illusory.

We've used the Ann and Beth case to raise a worry for Deference, but one can also use this case to raise a worry for General Deference. Indeed, we don't even need to modify the argument to do so - for if Evidential Inputs is true (something that General Deference allows) then the we can run precisely the same argument against General Deference. (Alternatively, if we don't want to assume Evidential Inputs, then we can modify the argument by replacing the assumption that you believe Ann has strictly more evidence than you, and that Beth has strictly more evidence than both you and Ann, with the assumption that you believe Ann is strictly better than you with respect to ranked inputs, and Beth is strictly better than both of you with respect to ranked inputs.) But this argument is ultimately not compelling, for reasons we've already seen. Namely, once we examine these purported reductios in more detail, we find that the cases they appeal to in order to obtain these absurd results describe situations that are impossible.

\section{Expert Principles}

Greco \& Hedden's (2016) Deference is a principle governing rational deference for subjects whose doxastic attitudes consist of beliefs. But one might also want a principle governing rational deference for subjects whose doxastic attitudes consist of credences. In the literature, such principles are known as "expert principles". Expert principles relate the credences

\footnotetext{
${ }^{24}$ This is a reductio of the Ann and Beth-based argument against Deference sketched in the text. One can try to modify the case in various ways to revive the Ann and Beth-style argument. But it's hard to find a straightforward way to do this. Perhaps the easiest way to get a feel for how hard this is to try one's hand at constructing concrete examples (with the details filled in) that leads Deference to make the desired prescriptions, and seeing how they fall through. But I'll briefly describe a few of the potential moves here, and why they don't work.

One way to try to revive the Ann and Beth-based argument against Deference, and maintain that believing $\operatorname{Def}_{\mathrm{Ann}} \wedge \operatorname{Def}_{\text {Beth }}$ could be justified after all, is to consider a case in which even though your total evidence $E$ justifies you in believing that Beth permissibly believes $\neg X$, you believe Ann's extra evidence $F$ makes it the case that Ann isn't justified in believing that Beth permissibly believes $\neg X$. In such a case, it might seem that Ann is justified in believing $X$ after all. But it's not clear this move works. For suppose your evidence does make you justified in believing that Ann's extra evidence $F$ makes it the case that Ann isn't justified in believing that Beth permissibly believes $\neg X$. Then it seems your evidence would also fail to justify your believing that Beth permissibly believes $\neg X$, and again we have a reductio of the claim that you could be justified in believing $\operatorname{Def}_{\mathrm{Ann}} \wedge \operatorname{Def}_{\mathrm{Beth}}$.

Another way to try to revive the Ann and Beth-style argument against Deference is to consider a slightly different case, where you believe both Ann and Beth have strictly more evidence than you, but neither has strictly more evidence than each other. (E.g., perhaps you have total evidence $E$, Ann has total evidence $E \wedge F$, and Beth has total evidence $E \wedge G$.) But, again, it's not clear that your evidence could justify you in believing Ann and Beth have such evidence and permissibly believe $X$ and $\neg X$, respectively. For Ann and Beth have all of your evidence, and so know about each other's beliefs. How this shakes out depends on the details of what this evidence is, but one could plausibly maintain that no matter how you flesh out these details, something will break - either Ann's evidence will fail to justify her in believing $X$, or Beth's evidence will fail to justify her in believing $\neg X$, or both.
} 
of rational subjects to the credences of those they believe to be epistemic experts relative to themselves. ${ }^{25}$

Levinstein (2015) has recently presented (though not endorsed) an argument for Unique Outputs that appeals to expert principles. Although this argument differs from Greco and Hedden's, the two arguments share some key features. And just as a careful look at Greco and Hedden's argument allowed us to draw some conclusions about the right form of beliefbased deference principles, Levinstein's argument allows us to draw some conclusions about the right form of expert principles.

Let $\stackrel{R}{R}$ be the set of rational (i.e., permissible) initial credence functions. Levinstein's argument begins by suggesting that the following is entailed by a plausible expert principle:

Weak Perm Expert (WPE): There exists a probability function $b$ and proposition $E$ such that: for every probability function $r$, if you have credences $b$ and total evidence $E$, you're obligated to be such that $b(\cdot \mid r \in \stackrel{\circ}{R})=r(\cdot \mid r \in \stackrel{\circ}{R} \wedge E) .{ }^{26,27}$

WPE is much weaker than one would like a full-fledged expert principle to be, of course; it just makes the existential claim that some credence function and evidence pair should be deferential in the manner described. But WPE is all Levinstein needs by way of an expert principle to set up his argument.

Let $r$ and $r^{\prime}$ be probability functions. Suppose you have credences $b$ and total evidence $E$.

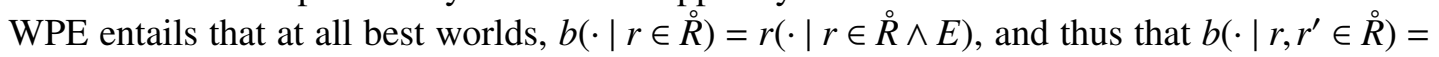
$r\left(\cdot \mid r, r^{\prime} \in \stackrel{\circ}{R} \wedge E\right)$. Likewise, WPE entails that at all best worlds, $b\left(\cdot \mid r^{\prime} \in \stackrel{\circ}{R}\right)=r^{\prime}\left(\cdot \mid r^{\prime} \in \stackrel{\circ}{R} \wedge E\right)$,

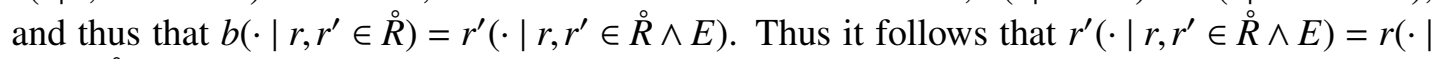

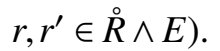

So given WPE, it seems $r$ and $r^{\prime}$ must assign the same values conditional on $E$ and each other being permissible. That is, it looks like mutual knowledge of the permissibility of each other's credences forces them to agree (given E). Now, this doesn't entail that Permissive

\footnotetext{
${ }^{25}$ There is a large literature about such principles, starting with Gaifman (1988); see Pettigrew \& Titelbaum (2014) for recent discussion and references. Most of the discussion in this literature focuses on how one should defer to an "expert". Of special interest, given the kinds of deference principles being discussed here, are discussions which avoid the (sometimes vague) notion of an "expert", and instead focus directly on the link between what a subject believes to be rational and what is rational for the subject. For discussions of these kinds of principles, see Christensen (2010), Elga (2013), and Levinstein (2015).

${ }^{26}$ Levinstein (2015) formulates WPE as follows (p21):

Weak Perm Expert: "For some $b$ with total evidence $E$ and for all probability functions $r, b(\cdot \mid r \in \stackrel{R}{R})=r(\cdot \mid E \wedge r \in \stackrel{\circ}{R})$." (Note that since $b$ is just a function and $E$ a proposition, there needs to be an implicit "if you have credences $b$ and total evidence $E$, then" clause before the equation in order for the norm to apply to particular subjects.) This statement of the principle leaves the location of the deontic operator that gives it normative force implicit, and there are two natural places one might take it to be. First, one might adopt a narrow scope reading of the principle, and take there to be an implicit obligation operator that applies just to the formula in question. This is to read the principle as saying something like "there's a $b$ and $E$ such that, for all $r$, if you have credences $b$ and evidence $E$, then your credences should satisfy the following equation". Second, one might adopt a wide scope reading of the principle, and take there to be an implicit obligation operator that holds over the entire conditional. This is to read the principle as saying something like "there's a $b$ and $E$ such that, for all $r$, you ought to be such that if you have credences $b$ and evidence $E$, then your credences satisfy the following equation". In the text I've adopted the narrow scope reading because Levinstein's argument for Unique Outputs requires it (cf. footnote 28).

${ }^{27}$ Following Levinstein, I'll leave implicit the assumptions needed to ensure that all of the conditional probabilities mentioned in the formulation of WPE and the argument discussed below are well-defined.
} 
Outputs is false - for example, one could maintain that there are multiple permissible doxastic states available to subjects when they're unsure about what credence functions are permissible. But, following Levinstein, let's grant that plausible versions of Permissive Outputs should allow for subjects with different permissible credence functions to still disagree after learning that both of their credence functions are permissible. If so, then this result suggests that no plausible version of Permissive Outputs is true; i.e., that WPE gives us reason to believe Unique Outputs. ${ }^{28,29}$

Although this is an interesting argument, it's not compelling because WPE is implausible. Like Deference, WPE employs mismatching operators. Roughly, WPE says that if you have $b$ and $E$, it's obligatory to line up your credences with functions you think are permissible. But it's implausible to maintain that it's obligatory to line up your credences with the credence functions you think are permissible; after all, you might think several different and incompatible credence functions are permissible. ${ }^{30}$

Indeed, given how WPE is formulated, these mismatching operators lead WPE to make inconsistent prescriptions. Let $r$ and $r^{\prime}$ be a pair of distinct probability functions such that $r\left(r, r^{\prime} \in \stackrel{\circ}{R} \wedge E\right)=r^{\prime}\left(r, r^{\prime} \in \stackrel{\circ}{R} \wedge E\right)=1$. WPE entails that at all best worlds, $b(\cdot \mid r \in \stackrel{\circ}{R})=r(\cdot \mid r \in$

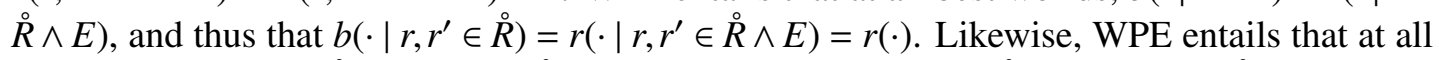

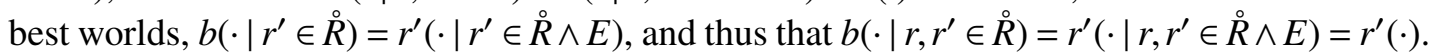
But since $r \neq r^{\prime}$, we can't consistently require $b\left(\cdot \mid r, r^{\prime} \in \stackrel{R}{R}\right)$ to be equal to both $r(\cdot)$ and $r^{\prime}(\cdot)$, so these prescriptions are inconsistent. And these inconsistent prescriptions would not arise if WPE had matching operators. ${ }^{31}$

\footnotetext{
${ }^{28}$ Note that this argument requires a narrow scope understanding of WPE (cf. footnote 26). For a wide scope understanding of WPE won't entail that at all best worlds $b(\cdot \mid r \in \stackrel{\circ}{R})=r(\cdot \mid r \in \stackrel{\circ}{R} \wedge E)$, it'll just entail that at all best worlds either (a) $b(\cdot \mid r \in \stackrel{\circ}{R})=r(\cdot \mid r \in \stackrel{\circ}{R} \wedge E$ ) or (b) you don't have credences $b$ and total evidence $E$. And this disjunctive requirement won't allow us to derive $r\left(\cdot \mid r, r^{\prime} \in \stackrel{\circ}{R} \wedge E\right)=r^{\prime}\left(\cdot \mid r, r^{\prime} \in \stackrel{\circ}{R} \wedge E\right)$. After all, this disjunctive requirement is compatible with (a) never obtaining.

${ }^{29}$ This is a slightly tweaked version of Levinstein's argument. The original argument attempted to derive $r\left(\cdot \mid r, r^{\prime} \in\right.$ $\stackrel{\circ}{R})=r^{\prime}\left(\cdot \mid r, r^{\prime} \in \stackrel{\circ}{R}\right)$, allowing one to avoid any reference to $E$. However, this conclusion doesn't seem to follow from WPE. (In correspondence, Levinstein has suggested that his preferred way of getting around this problem would be to try to modify WPE.) In any case, the differences between these two versions of the argument don't matter for our purposes.

${ }^{30}$ As we saw in section 3.2, given a wide scope principle like Deference, one can try to avoid these implausible consequences by adopting the (question begging) assumption that all rational subjects believe Unique Outputs, and so will only ever think one doxastic state is permissible. But even that (question begging) option isn't available here, since WPE is a narrow scope principle (cf. footnote 28) that makes prescriptions based on a subject's actual doxastic state. Thus WPE entails that a (possibly irrational) subject who believes several different credence functions are permissible is obligated to line up their credences with all of them. And WPE has this consequence regardless of whether rational subjects believe Unique Outputs or not.

31 If WPE employed matching permission operators, then it wouldn't yield inconsistent prescriptions because instead of requiring $b\left(\cdot \mid r, r^{\prime} \in \stackrel{\circ}{R}\right)$ to equal $r(\cdot)$ and $r^{\prime}(\cdot)$, it would merely allow $b\left(\cdot \mid r, r^{\prime} \in \stackrel{\circ}{R}\right)$ to equal $r(\cdot)$ or $r^{\prime}(\cdot)$. And if WPE employed matching obligation operators, and so applied constraints on $b$ conditional on $r$ being obligatory, then it wouldn't yield inconsistent prescriptions because there wouldn't be any probability functions $r$ and $r^{\prime}$ which satisfy the conditions the derivation requires; i.e., which assign a value of 1 to both $r$ and $r^{\prime}$ being obligatory.

In correspondence, Levinstein has suggested that his preferred way of getting around the conflicting prescriptions issue would be to modify WPE in certain ways (such as by restricting the probabilistic $r$-functions WPE makes claims about). These changes alone won't change the fact that WPE employs mismatching operators, however, so these changes alone still leave us with an implausible principle. (That said, Levinstein has also expressed sympathy for
} 
If we modify WPE so that its operators match then we can avoid these implausible consequences. But once we make these changes the WPE-based argument for Unique Outputs will no longer go through. For the WPE-based argument for Unique Outputs, like the Deferencebased argument for Evidential Uniqueness, crucially relies on these mismatching operators.

Let's see why the WPE-based argument for Unique Outputs won't work given matching operator versions of WPE. Suppose we modified WPE so that it employed matching permission

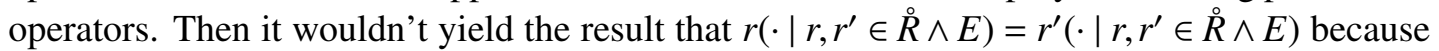
it would merely allow (instead of require) $b\left(\cdot \mid r, r^{\prime} \in \stackrel{R}{\text { ) }}\right.$ ) to be equal to $r\left(\cdot \mid r, r^{\prime} \in \stackrel{R}{R} \wedge E\right.$ ) or $r^{\prime}\left(\cdot \mid r, r^{\prime} \in \stackrel{\circ}{R} \wedge E\right)$.

Suppose instead that we modified WPE so that it employed matching obligation operators. Let $O$ be the set containing the obligatory initial credence function. If WPE employed match-

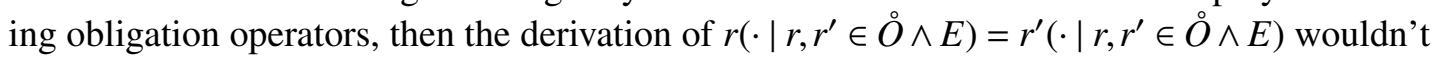
work because none of these conditional probabilities would be well-defined (since if $r \neq r^{\prime}$, it's impossible for both $r$ and $r^{\prime}$ to be obligatory). ${ }^{32,33}$

So ultimately, like the deference principles discussed in sections 3 and 4, WPE and its variants fail to provide us with a reason to adopt Unique Outputs.

In section 4 I formulated a general deference principle for subjects whose doxastic attitudes consist of beliefs. I won't try to formulate an analogous general expert principle for subjects whose doxastic attitudes consist of credences. In part this is because the move to credences raises a number of complications I don't have space to address, and in part this is because I don't know how to formulate such a principle so that it can plausibly apply to any normative realm, as General Deference does (cf. footnote 18). But we don't need to pursue this more ambitious project to draw some conclusions about the form of a satisfactory expert principle. Namely, we should expect any satisfactory expert principle that links a subject's credences with the credences the subject takes to be permissible or obligatory to employ matching deontic operators.

\section{Conclusion}

Consider the normative realms for which you think the notions of permission and obligation require a subjectivist theory - a theory whose prescriptions are mediated by a subject's doxastic state. For these realms, it's natural to maintain that there's some kind of link between a subject's beliefs about what's permissible or obligatory and what is in fact permissible or obligatory for them. That is, it's natural to endorse some kind of deference principle.

I've suggested that we should expect a general deference principle - one which doesn't

modifying WPE so that its operators match.)

${ }^{32}$ Note that the point being made in these two paragraphs is different from the point being made in footnote 31. Footnote 31 spells out why the matching-operator variants of WPE, unlike WPE, won't yield conflicting prescriptions. The two paragraphs above spell out why the matching-operator variants of WPE, unlike WPE, won't yield an argument for Unique Outputs.

${ }^{33}$ We've been following Levinstein in avoiding notational clutter by leaving implicit all of the assumptions required to ensure that the relevant conditional probabilities are well-defined (cf. footnote 27). But we can't make these implicit

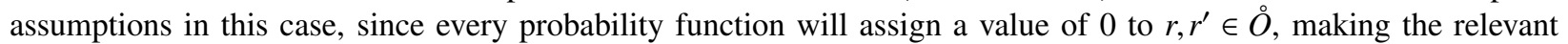
conditional probabilities undefined. 
presuppose a particular account of what factors are normatively relevant, and which applies to all subjectivist normative realms - to look like this:

General Deference: For any $X$ and subject $S$, it's permissible [obligatory] to be such that: if you believe that it's permissible [obligatory] for $S$ to $X$, and believe the normatively relevant facts are either the same for you and $S$, or differ only regarding normatively relevant facts for which $S$ is in a strictly better position, then you $X$.

And I've shown how one can motivate such a principle using some natural anti-akratic principles: namely, that it's permissible [obligatory] for you to be such that if you believe $X$ is permissible [obligatory] for you, then you $X$.

This still leaves us with some interesting questions regarding whether and how to formulate more precise versions of this principle in particular contexts (e.g., when focusing on the epistemic realm and subjects whose doxastic attitudes consist of credences). But here too our earlier discussion provides some helpful guidance with respect what these principles should look like. For example, we should expect any plausible quantitative deference principle to employ matching deontic operators.

Recently, Greco \& Hedden (2016) and Levinstein (2015) have shown how one might employ certain kinds of deference principles in order to argue for either Evidential Uniqueness or Unique Outputs. I've suggested that these arguments are not compelling, because the deference principles they employ are implausible. And if we adopt more plausible deference principles, these arguments won't work. So, ultimately, there's little reason to think that deference considerations provide a compelling reason to adopt Evidential Uniqueness or Unique Outputs. ${ }^{34}$

\section{Appendix}

Let's see how we can use the following anti-akratic principles:

Anti-Akratic Principle (Permission) (AAPP): For any proposition $X$, it's permissible to be such that if you believe $X$ is permissible, then you $X$.

Anti-Akratic Principle (Obligation) (AAPO): For any proposition $X$, it's obligatory to be such that if you believe $X$ is obligatory, then you $X$.

to derive General Deference (Same Inputs), which consists of two conjuncts:

General Deference (Same Inputs, Permission) (GDSIP): For any $X$ and subject $S$, it's permissible to be such that: if you believe that it's permissible for $S$ to $X$, and believe that the normatively relevant facts are the same for you and $S$ (i.e., believe that the inputs of $f$ are the same for you and $S$ ), then you $X$.

General Deference (Same Inputs, Obligation) (GDSIO): For any $X$ and subject $S$, it's obligatory to be such that: if you believe that it's obligatory for $S$ to $X$, and believe that the normatively relevant facts are the same for you and $S$ (i.e., believe that the inputs of $f$ are the same for you and $S$ ), then you $X$.

\footnotetext{
${ }^{34}$ I'd like to thank Maya Eddon, Daniel Greco, Brian Hedden, Ben Levinstein, Alejandro Perez-Carballo, and Jonathan Vogel for helpful comments and discussion.
} 
GDSIP and GDSIO might seem to follow trivially from AAPP and AAPO. To some extent that's true. But while the derivation of the narrow scope variants of GDSIP and GDSIO from narrow scope variants of AAPP and AAPO is straightforward, the derivation of the actual (wide scope) principles GDSIP and GDSIO from the actual (wide scope) principles AAPP and AAPO takes a bit more work.

Two preliminary comments. First, these derivations assume that, at best worlds, one's beliefs are closed under logical and analytic entailment. ${ }^{35}$ Second, instead of constantly repeating "for any $X$ ", I'll streamline the discussion by leaving the universal quantifier over $X$ implicit in what follows.

Let's start by showing how to derive GDSIP from AAPP. Note that (at any best world) if you believe $X$ is permissible for you, then you believe that there is a subject (you) with the same inputs for whom $X$ is permissible. Likewise (at any best world) if you believe that there is a subject $S$ with the same inputs as you for whom $X$ is permissible, then you believe $X$ is permissible for you. Thus the following biconditional holds: (it's permissible to be such that if you believe you can permissibly $X$, then you $X$ ) iff (it's permissible to be such that if you believe that there's a subject $S$ with the same inputs who can permissibly $X$, then you $X$ ). That is, AAPP is true iff:

(A) There's a best world at which the following conditional is true: if you believe there's a subject $S$ that has the same inputs as you who can permissibly $X$, then you $X$.

And (A) entails (B), a reformulation of GDSIP:

(B) For any subject $S$, there's a best world where the following conditional is true: if you believe $S$ has the same inputs as you and can permissibly $X$, then you $X$.

We can see that (A) entails (B) by showing that the falsity of (B) entails the falsity of (A) and applying contraposition. For (B) to be false, there must be an $S$ such that there's no best world where if you believe $S$ has the same inputs as you and can permissibly $X$, then you $X$; i.e., there must be an $S$ such that, at all best worlds, you believe $S$ has the same inputs as you and can permissibly $X$, and you don't $X$. But that entails that at all best worlds, you believe that there's a subject $S$ with the same inputs who can permissibly $X$, and you don't $X$. And that entails that (A) is false.

Since AAPP is true iff (A), and (A) entails GDSIP, it follows that AAPP entails GDSIP.

Now let's look at how to derive GDSIO from AAPO. Note that (at any best world) if you believe $X$ is obligatory for you, then you believe that there is a subject (you) with the same inputs for whom $X$ is obligatory. Likewise (at any best world) if you believe that there is a subject $S$ with the same inputs as you for whom $X$ is obligatory, then you believe $X$ is obligatory for you. Given this, the following biconditional holds: (it's obligatory to be such

\footnotetext{
${ }^{35}$ While this assumption is relatively uncontroversial for epistemically best worlds (and thus for deriving General Deference (Same Inputs) for epistemic permission and obligation), it's less clear why it's plausible with respect to, say, prudentially or morally best worlds. I think this assumption is still tenable in those cases given the kind of "subjectivist" approach motivating AAPP and AAPO. Because this approach incorporates features of a subject's epistemic life into its prescriptions, it naturally takes on features of epistemic norms that would not show up on an "objectivist" approach. In any case, those concerned about this assumption can either take the fact that one can derive General Deference (Same Inputs) for subjects whose beliefs are closed under logical and analytic entailment to merely provide a motivation for (instead of a derivation of) General Deference (Same Inputs), or restrict General Deference (Same Inputs) to subjects whose beliefs are closed under logical and analytic entailment.
} 
that if you believe that you're obligated to $X$, then you $X$ ) iff (it's obligatory to be such that if you believe that there is a subject $S$ with the same inputs as you who is obligated to $X$, then you $X$ ). That is, AAPO is true iff:

(C) At all best worlds the following conditional is true: if you believe there is a subject $S$ with the same inputs as you for whom $X$ is obligatory, then you $X$.

And (C) entails (and, in fact, is equivalent to) (D), a reformulation of GDSIO:

(D) For every subject $S$, at all best worlds, the following conditional is true: if you believe $S$ has the same inputs as you and that $X$ is obligatory for $S$, then you $X$.

We can see that (C) entails (D) by showing that the falsity of (D) entails the falsity of (C) and applying contraposition. For (D) to be false, there must be an $S$ such that, at some best world, you believe $S$ has the same inputs as you and is obligated to $X$, but you don't $X$. This entails that there is some best world at which you believe there is some $S$ with the same inputs as you who is obligated to $X$, but you don't $X$. And that is precisely what needs to be the case in order for $(\mathrm{C})$ to be false.

(To see that (C) and (D) are in fact equivalent, note that the entailment also goes the other way. For (C) to be false, there must be some best world at which you believe there is a subject $S$ with the same inputs as you who is obligated to $X$, but you don't $X$. But that entails that there's some $S$ such that, at some best world, you believe $S$ has the same inputs as you and is obligated to $X$, but you don't $X$, and thus entails that (D) is false.)

Since AAPO is true iff (C), and (C) entails (and is in fact equivalent to) GDSIO, it follows that AAPO entails GDSIO.

\section{References}

Ballantyne, N., \& Coffman, E. J. (2011). Uniqueness, evidence, and rationality. Philosophers' Imprint, 11(18).

Christensen, D. (2010). Rational reflection. Philosophical Perspectives, 24(1), 121-140.

Davidson, D. (1970). How is weakness of the will possible? In J. Feinberg (Ed.) Moral Concepts. Oxford University Press.

Dogramaci, S., \& Horowitz, S. (2016). An argument for uniqueness about evidential support. Philosophical Issues, 26(1), 130-147.

Elga, A. (2013). The puzzle of the unmarked clock and the new rational reflection principle. Philosophical Studies, 164(1), 127-139.

Fantl, J., \& McGrath, M. (2009). Knowledge in an Uncertain World. Oxford University Press.

Gaifman, H. (1988). A theory of higher order probabilities. In B. Skyrms, \& W. Harper (Eds.) Causation, Chance, and Credence. Kluwer.

Greco, D. (2014). A puzzle about epistemic akrasia. Philosophical Studies, 167(2), 201-219. 
Greco, D., \& Hedden, B. (2016). Uniqueness and metaepistemology. Journal of Philosophy, 113(8), 365-395.

Horowitz, S. (2014). Epistemic akrasia. Noûs, 48(4), 718-744.

Kelly, T. (2013). Evidence can be permissive. In M. Steup, \& J. Turri (Eds.) Contemporary Debates in Epistemology, (p. 298). Blackwell.

Kratzer, A. (2012). Modals and Conditionals: New and Revised Perspectives. Oxford University Press.

Levinstein, B. A. (2015). Permissive rationality and sensitivity. Philosophy and Phenomenological Research, 92(1).

Matheson, J. (2011). The case for rational uniqueness. Logic and Episteme, 2(3), 359-373.

McNamara, P. (2010). Deontic logic. In E. Zalta (Ed.) Stanford Encyclopedia of Philosophy.

Meacham, C. J. G. (2013). Impermissive bayesianism. Erkenntnis, (S6), 1-33.

Peels, R., \& Booth, A. R. (2014). Why responsible belief is permissible belief. Analytic Philosophy, 55(1), 75-88.

Pettigrew, R., \& Titelbaum, M. G. (2014). Deference done right. Philosophers' Imprint, 14(35), 1-19.

Podgorski, A. (2016). Dynamic permissivism. Philosophical Studies, 173(7), 1923-1939.

Schoenfield, M. (2013). Permission to believe: Why permissivism is true and what it tells us about irrelevant influences on belief. Noûs, 47(1), 193-218.

Stich, S. (1993). The Fragmentation of Reason: Preface to a Pragmatic Theory of Cognitive Evaluation. MIT Press.

Stroud, S. (2014). Weakness of will. In E. Zalta (Ed.) Stanford Encyclopedia of Philosophy.

Titelbaum, M. G., \& Kopec, M. (manuscript). Plausible permissivism.

vonFintel, K. (2011). Conditionals. In C. M. Klaus von Heusinger, \& P. Portner (Eds.) Semantics: An international handbook of meaning, vol. 2, (p. 1515?1538).

White, R. (2005). Epistemic permissiveness. Philosophical Perspectives, 19(1), 445-459. 